Meta

Journal des traducteurs

Translators' Journal

\title{
Les organismes de terminologie du monde francophone et les organismes internationaux de terminologie
}

\section{Nycole Bélanger}

Volume 22, numéro 2, juin 1977

URI : https://id.erudit.org/iderudit/002045ar

DOI : https://doi.org/10.7202/002045ar

Aller au sommaire du numéro

Éditeur(s)

Les Presses de l'Université de Montréal

ISSN

0026-0452 (imprimé)

1492-1421 (numérique)

Découvrir la revue

Citer cet article

Bélanger, N. (1977). Les organismes de terminologie du monde francophone et les organismes internationaux de terminologie. Meta, 22(2), 126-132.

https://doi.org/10.7202/002045ar d'utilisation que vous pouvez consulter en ligne. 


\section{LES ORGANISMES DE TERMINOLOGIE DU MONDE FRANCOPHONE ET LES ORGANISMES INTERNATIONAUX DE TERMINOLOGIE}

1. Au hasard des lectures, des exposés ou des conversations, traducteurs et terminologues apprennent l'existence d'organismes de terminologie. Toutefois, souvent ils n'en connaissent que le nom, si ce n'est que le sigle. Ces organismes sont mal connus, et davantage encore leur situation les uns par rapport aux autres. Nous allons donc tenter d'esquisser un schéma simple des principaux organismes ayant une activité terminologique dans le monde francophone. Puis, nous présenterons les organismes internationaux de terminologie avec lesquels ils sont susceptibles d'être en relation.

6. Grand Larousse Encyclopédique, p. 111.

7. Peter Cotes, op. cit., p. 8. 


\section{Au pays}

Au Canada, deux organismes principaux exercent une activité terminologique : le Bureau des traductions du gouvernement fédéral et la Régie de la langue française du Québec.

2.1 Créé il y a une douzaine d'années, le Centre de termino'ogie d'Ottawa était déjà bien connu par la publication de ses nombreux Bulletins de terminologie et par son bulletin d'information mensuel, l'Actualité terminologique. En 1975, il s'est donné de nouvelles structures et un nouveau nom: Direction générale de la terminologie et de la documentation ${ }^{\text {? }}$.

Dans l'ensemble, les fonctions de cette unité administrative restent les mêmes, soit établir ou recueillir l'information terminologique, la traiter, la normaliser et la diffuser dans les sections du Bureau des traductions dont elle fait partie et dans les services de l'État. La nouvelle structure permettra de faire face aux besoins grandissants en matière de terminologie.

Par ailleurs, le Bureau des traductions utilise désormais le logiciel de la Banque de terminologie de l'Université de Montréal. Il s'est ainsi donné un outil puissant, à la mesure de son immense tâche.

En février 1976, la direction a tenu un colloque canadien sur les fondements d'une méthodologie générale de la recherche et de la normalisation en terminologie et en documentation.

2.2 Au Québec, la Régie de la langue française (autrefois, l'Office de la langue française) comporte une direction de la terminologie dont les fonctions sont de faire des recherches terminologiques, d'établir des méthodes de recherche en terminologie et de coordonner les travaux de terminologie en cours au Québec. Les secteurs d'activité de la direction de la terminologie sont multiples : alimentation, automobile, activités industrielles, gestion, terminologie juridique, etc., sans compter les secteurs affectés particulièrement à la néologie de langue technique et à la néologie en langue commune.

À ce jour, nombreux sont les lexiques publiés par la R.L.F. En outre, une nouvelle version du Guide de travail en terminologie doit paraître sous peu.

La R.L.F. a organisé quatre colloques internationaux portant sur la terminologie : les données terminologiques (1972), la normalisation linguistique (1973), la néologie (1974) et un essai de définition de la terminologie (1975). En janvier 1976, elle a réuni les terminologues du Québec qui ont fait le point sur leurs méthodes de travail.

La R.L.F. met présentement sur pied une banque de terminologie dont une partie est consacrée à un dictionnaire automatisé (TERMINOQ I), l'autre à l'in-

1. P.-E. Larose, « Réorganisation des services terminologiques du Bureau des traductions ", l'Actualité terminologique (Bulletin mensuel de la direction de la terminologie et de la documentation, Bureau des traductions, Secrétariat d'Etat, Ottawa) vol. 9, nos 1 et 2 , janvier et février 1976. 
ventaire des ressources terminologiques existantes au pays et à l'étranger (TERMINOQ II).

En attendant la mise au point du système informatique, la direction de la terminologie a édité un certain nombre de microfiches ou microfilms disponibles sur demande ainsi qu'un Inventaire des travaux de terminologie publiés ${ }^{2}$ et un Inventaire des travaux de terminologie en cours ${ }^{3}$.

2.3 Le service de linguistique de Radio-Canada joue un rôle important en matière de terminologie au pays. Ses fiches linguistiques et son bulletin $C^{\prime} e s t-\grave{a}$-dire ont acquis leurs lettres de noblesse depuis fort longtemps. Le service a aussi à son actif plusieurs vocabulaires touchant principalement la radio, la télévision et la gestion.

2.4 Depuis quelques années maintenant, les services de traduction des entreprises du Québec se dotent de terminologues (Bell Canada, CN/Air Canada, CIL, Hydro-Québec, IBM, etc.). On ne peut évidemment considérer ces unités comme des organismes de terminologie. Toutefois, chacun de ces groupes a développé une compétence particulière dans son secteur d'activité et de ce fait a un rôle important à jouer dans le réseau des échanges terminologiques.

\section{3. À l'êtranger}

\subsection{France}

3.1.1 Le Haut Comité de la langue française a été créé par décret en 1966 pour conseiller le gouvernement. Depuis 1972, il a mis sur pied plusieurs commissions ministérielles de terminologie (pétrole, énergie nucléaire, transports, audiovisuel, techniques spatiales, bâtiment, informatique, économie et finances, santé) dont les travaux sont publiés au Journal afficiel de la République française sous forme d'arrêtés. Ces arrêtés comportent toujours deux listes de termes. Les termes de la liste no 1 sont d'emploi obligatoire pour l'administration publique. Les termes de la liste $n^{\circ} 2$ sont recommandés et en quelque sorte soumis à l'enquête publique.

Au Québec, 1a Régie de la langue française adopte ipso facto les termes de la liste no 1.

3.1.2 De création toute récente, l'AFTERM ou Association française de terminologie a vu le jour sous les auspices du Haut Comité. Sa mission est de coordonner l'activité des groupes de terminologie du secteur privé en France et de provoquer un plus grand intérêt pour les questions de terminologie dans l'entreprise française. Elle a tenu en juin 1976 un colloque international portant sur l'élaboration et l'emploi des terminologies scientifiques et techniques.

2. Québec - Office de la langue française, Inventaire des travaux de terminologie, Québec, Ministère de l'Education, $1973,152 \mathrm{p}$.

3. Québec $\rightarrow$ Office de la langue française, Inventaire des travaux de terminologie - Projets et travaux en cours connus au 31 août 1974, Québec, éditeur officiel, octobre 1974, $61 \mathrm{p}$. 
3.1.3 L'AFNOR (Association française de normalisation) est l'organisme officiel de normalisation en France. Elle publie des normes dans les domaines les plus divers : métallurgie, mécanique, électronique, énergie nucléaire, etc.

Le travail terminologique des commissions de I'AFNOR a un prolongement très important sur le plan international. En effet, c'est l'AFNOR qui fournit aux comités et aux sous-comitćs de l'ISO (dont nous parlerons plus loin) les termes techniques français relatifs à leurs travaux.

En vue du traitement automatique du vocabulaire issu des normes françaises et internationales, l'AFNOR a entrepris en 1972 la mise sur pied d'une banque de données terminologiques (NORMATERM).

\subsection{Belgique}

Le Bureau de terminologie de la Commission des Communautés européennes à Bruxelles existe depuis 1958. Il œuvre dans tous les secteurs d'activités de la Commission des Communautés européennes. Il publie de nombreux lexiques multilingues et un Bulletin de la traduction.

\subsection{Luxembourg}

3.3.1 Le Bureau de terminologie des Communautés européennes au Luxembourg a été créé en 1963. Il prépare de nombreux lexiques multilingues dont les sujets sont très variés : utilisation de l'acier, sécurité et hygiène du travail, statistiques, sciences de l'information, etc. Un Bulletin de terminologie rend compte périodiquement de ses activités et de l'acutalité terminologique en général.

Enfin, le Bureau exploite depuis quelques années déjà une banque de terminologie.

3.3.2 Créé en 1965, le Bureau de terminologie du parlement européen publie régulièrement des lexiques sur des sujets d'actualité (transports, droits de l'homme, marché des capitaux, etc.). Ces lexiques sont généralement fondés sur l'analyse de documents officiels : traités, règlements, recommandations, directives émanant du parlement européen. Le Bureau publie aussi un bulletin intitulé Informations terminologiques.

\section{Au plan international}

4.1 Le Conseil international de la langue française (CILF), dont le siège est à Paris, a été créé en 1967 à l'occasion de la deuxième Biennale de la langue française qui se tenait à Québec. Il a pour tâche de maintenir l'unité du français dans le monde.

À cette fin, il publie des vocabulaires techniques, rédige des manuels techniques destinés aux pays du Tiers-Monde et collabore à la rédaction de thésaurus documentaires. Il publie une revue de terminologie intitulée la Banque des mots et offre un service mensuel de fiches terminologiques intitulé la Clé des mots. 
Le Conseil groupe actuellement des représentants de vingt-quatre pays entièrement ou partiellement de langue française.

4.2 L'Organisation internationale de normalisation (ISO) déploie une grande activité terminologique et c'est à ce titre que nous la considérons.

L'ISO groupe les organismes de normalisation de soixante-treize pays. Son activité consiste à établir des normes internationales en vue de faciliter la collaboration entre les pays dans les domaines intellectuel, scientifique, technologique et économique. La normalisation terminologique étant inhérente à la normalisation des concepts, les questions de terminologie ont une importance capitale au sein des nombreux comités techniques (les TC - Technical Committees).

Certains documents de l'ISO sont des lexiques. L'ISO compte aussi un comité technique particulièrement affecté aux principes relatifs à la terminologie, le TC 37 . Terminologie (principes et coordination). Ce comité se subdivise présentement en trois groupes de travail traitant respectivement des principes de la terminologie, de la présentation des vocabulaires et de l'aide que peut apporter l'informatique dans les travaux de terminologie et de lexicographie.

4.3 Infoterm (Centre international d'information pour la terminologie) a été fondé à Vienne en 1971. La création de cet organisme résulte d'une entente entre le Bureau de normalisation de Vienne et l'UNESCO.

Avec un effectif réduit, il a déjà publié The Road to Infoterm $^{4}$, recueil imposant de renseignements concernant les multiples aspects de la recherche terminologique. Un World Guide to Terminological Activities est actuellement sous presse.

Infoterm a tenu en avril 1975 un symposium sur la coopération internationale en terminologie. Cette réunion de spécialistes venus de tous les pays où il se fait de la recherche terminologique a permis de faire un autre pas vers l'établissement d'un réseau international d'information en terminologie.

\section{Conclusion}

Les relations qui existent entre les organismes de terminologie ne sont pas hiérarchisées; elles sont avant tout des relations d'information et elles relèvent autant, sinon plus, des contacts personnels que des ententes officielles. Les responsables de ces organismes se connaissent, se consultent. Les colloques nationaux et internationaux ont permis des échanges de vues extrêmement enrichissants en ce qui concerne les méthodes de recherche en terminologie et l'établissement de réseaux d'échange d'informations terminologiques. Bref, ces organismes sont liés par une volonté commune de progresser dans la connaissance de l'activité terminologique. De ce fait, il est permis de penser que nous pourrons arriver un jour à une réelle coordination des efforts dans ce domaine.

NyCOLE BÉLANGER

4. Eugen Wüster, The Road to Infoterm, Pullach bei München, Verlag Dokumentation, 1974, 145 p. 


\section{RÉSEAU DES ORGANISMES DE TERMINOLOGIE}

DU MONDE FRANCOPHONE ET

DES ORGANISMES DE TERMINOLOGIE INTERNATIONAUX

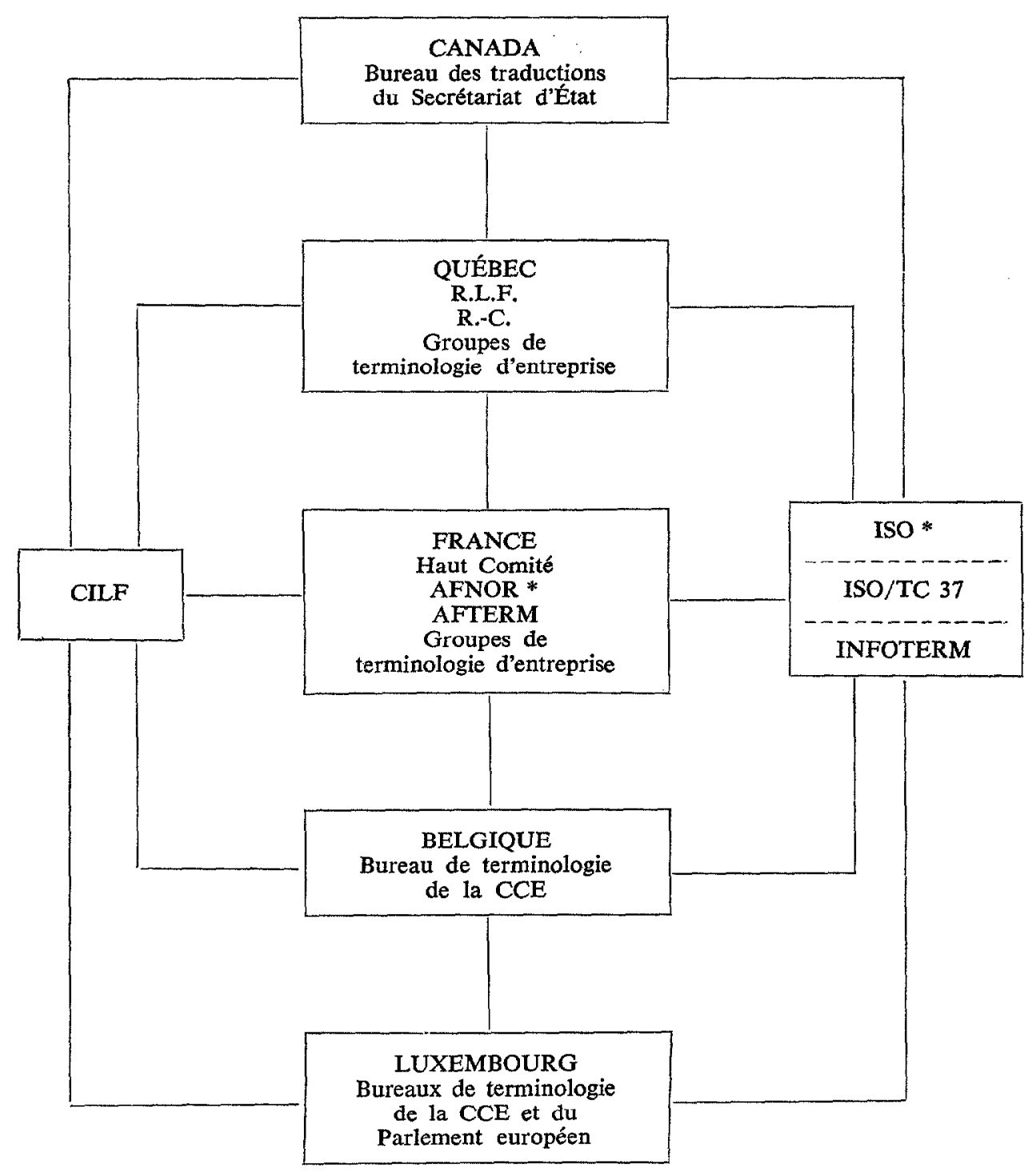

* L'AFNOR et l'ISO sont essentiellement des organismes de normalisation. Nous les mentionnons ici à cause de leur activité terminologique. 


\section{ADRESSES DES ORGANISMES}

\section{PAR ORDRE DE PRÉSENTATION DANS LE TEXTE}

Secrétariat d'État du Canada

Direction générale de la terminologie et de la documentation

Édifice Sir-Richard-Scott

591 , rue Laurier ouest

Ottawa (Ontario)

K1A 0 X3

Régie de la langue française Direction de la terminologie

700, boul. Saint-Cyrille est

Québec (Québec)

G1R 5A9

Radio-Canada

Service de linguistique

B.P. 6000

Montréal (Québec)

Haut Comité de la langue française

32, rue de Babylone

75700 Paris

FRANCE

Association française de terminologie (AFTERM)

Haut Comité de la langue française

32 , rue de Babylone

75700 Paris

FRANCF

Association française de normalisation

Tour Europe

Cedex 7

92080 Paris La Défense

FRANCE

Commission des Communautés européennes Bureau de terminologie

1, av. de Cortenbergh

1040 Bruxelles

BELGIQUE
Commission des Communautés européennes

Bureau de terminologie

Avenue Monterey (angle boul. Joseph 2)

LUXEMBOURG

Commission des Communautés européennes Bureau de terminologie du parlement européen

Case postale 1601

LUXEMBOURG

Conseil international de la langue française 105 ter, rue de Lille

75007 Paris

FRANCE

Organisation internationale de normalisation (ISO)

Secrétariat central

1 , rue de Varembé

Case postale 56

1211 Genève

SUISSE

Technical Committee 37

Österreichisches Normungsinstitut

Leopoldgasse 4

A-1021 Wien 2

AUTRICHE

Centre international d'information pour la terminologie (INFOTERM)

Österreichisches Normungsinstitut

Leopoldgasse 4

A-1021 Wien 2

AUTRICHE 